Pacific Journal of Mathematics

A NOTE ON THE GAUSS CURVATURE OF HARMONIC AND 


\section{A NOTE ON THE GAUSS CURVATURE OF HARMONIC AND MINIMAL SURFACES}

\section{GioRgio TALENTI}

We present some inequalities for the Gauss curvature of embedded surfaces in euclidean 3-space, which are either graphs of harmonic functions or minimal. The proofs exploit the following facts: (i) a partial differential equation constrains the curvature of the surfaces in question; (ii) the differential equation constrains in a significant way, via an isoperimetric inequality, the level lines of the curvature.

1. Introduction. Let $u$ be a (real-valued) harmonic function of two (real) variables $x$ and $y$, and let $K$ be the Gauss curvature of the graph of $u$. The following lemma is the starting point of our arguments.

LEMMA. The following equation

$$
K\left(K_{x x}+K_{y y}\right)-K_{x}^{2}-K_{y}^{2}=8 K^{3}
$$

holds (here subscripts stand for differentiation, e.g., $K_{x}=\partial K / \partial x$, etc.).

Proof. The Gauss curvature of the graph of $u$ is given by

$$
K=\left(1+u_{x}^{2}+u_{y}^{2}\right)^{-2}\left(u_{x x} u_{y y}-u_{x y}^{2}\right) .
$$

We have to show that formula (2) provides us with a kind of general solution of equation (1), i.e., formula (2) produces a solution to equation (1) whenever $u$ is a harmonic function.

Indeed, our harmonic function can be represented (at least locally) as the real part

$$
u(x, y)=\operatorname{Re} f(z)=\frac{1}{2}[f(z)+\overline{f(z)}]
$$

of some holomorphic function $f$ of the complex variable $z=x+i y$. Using (2) and (3) one easily finds the following alternative formula

$$
K=-\left(1+\left|f^{\prime}\right|^{2}\right)^{-2}\left|f^{\prime \prime}\right|^{2},
$$

where primes denote differentiation with respect to $z$.

The right-hand side of (4) involves a holomorphic function (namely $f^{\prime}$ ) and the first derivative of it. We want to eliminate this holomorphic function from (4) and equations involving deriva- 
tives of $K$. In other words, we seek a partial differential equation which is satisfied by any expression of the form

$$
K=-\left(1+|h|^{2}\right)^{-2}\left|h^{\prime}\right|^{2},
$$

where $h$ is an arbitrary holomorphic function. Such a differential equation is very close to one considered by Liouville [16] [17] and is easily found with the help of the complex derivatives

$$
\frac{\partial}{\partial z}=\frac{1}{2}\left(\frac{\partial}{\partial x}-i \frac{\partial}{\partial y}\right) \frac{\partial}{\partial \bar{z}}=\frac{1}{2}\left(\frac{\partial}{\partial x}+i \frac{\partial}{\partial y}\right) .
$$

Bearing in mind the usual rules (e.g., $\partial h / \partial z=h^{\prime}, \partial \bar{h} / \partial z=0, \partial h / \partial \bar{z}=0$, $\partial \bar{h} / \partial \bar{z}=\bar{h}^{\prime}$ where $h$ is any holomorphic function of $z=x+i y$ ), in few steps one arrives at the following conclusion

$$
K \frac{\partial^{2} K}{\partial z \partial \bar{z}}-\frac{\partial K}{\partial z} \frac{\partial K}{\partial \bar{z}}=2 K^{3}
$$

an equivalent form of (1). The lemma is proved.

Observe that formula (4) implies the following property: $K$ is nonpositive and may have only isolated zeros. In a sense, this property is related to the maximum principle for harmonic functions: indeed, it tells us that the graph of $u$ cannot lie on one side of any of its tangent planes.

One aim of the present paper is to point out that equation (1) is a tool for deriving properties of $K$. In fact our results on the curvature of harmonic and minimal surfaces are inferred from the quality of such a curvature of being a nonpositive solution to a special second order partial differential equation, and are obtained by partially decoding the information comprised in that equation in an appropriate way. Thus our point of view coincides with a usual one in classical differential geometry, where differential equations often play the role of devices for storing information on geometric objects. Also, our investigations are similar in spirit to those on partial differential equations, made when the existence of solutions is obvious or taken for granted and a priori bounds for, or qualitative properties of solutions, are considered of primary interest.

By way of an example, let us prove the following property. The negative of $K$ cannot achieve a minimum at an interior point, unless the minimum is zero. In fact, suppose that $K$ is free from zeros. Then we can put

$$
-K=4 w^{-2}
$$


and equation (1) yields the equation

$$
w\left(w_{x x}+w_{y y}\right)=1+w_{x}^{2}+w_{y}^{2},
$$

showing that $w=2 / \sqrt{-K}$ is subharmonic.

As is well known, some a priori bounds for harmonic functions, and for combinations of their derivatives, automatically hold in the whole of the domain when the same bounds are available on the boundary. In fact, the maximum principle comes in whenever quantities, suitably related to those under estimation, are subharmonic functions. For instance, the above remark applies if the maximum of expressions such as

$$
\begin{aligned}
& u_{x}^{2}+u_{y}^{2}, \\
& u_{x x}^{2}+2 u_{x y}^{2}+u_{y y}^{2}, \\
& u_{x y}^{2}-u_{x x} u_{y y}, \cdots
\end{aligned}
$$

is to be estimated. Indeed, the expressions (8) obviously are subharmonic.

The last assertion can be strengthened. For one may check that each of the expressions (8) is a solution to the following equation

$$
\varphi\left(\varphi_{x x}+\varphi_{y y}\right)-\varphi_{x}^{2}-\varphi_{y}^{2}=0 .
$$

Of course, other more sophisticated objects might enrich the list (8), that share with the previous ones the property of significantly involving derivatives of $u$ and being solutions to equation (9). For the sake of completeness, let us quote

$$
H+i h,
$$

a complex-valued function having

$$
H=\left(1+u_{x}^{2}+u_{y}^{2}\right)^{-3 / 2}\left(\left(1+u_{y}^{2}\right) u_{x x}-2 u_{y} u_{x} u_{x y}+\left(1+u_{x}^{2}\right) u_{y y}\right),
$$

the mean curvature of the graph of $u$, and

$$
h=\left(1+u_{x}^{2}+u_{y}^{2}\right)^{-3 / 2}\left(u_{x} u_{y}\left(u_{x x}-u_{y y}\right)+\left(u_{y}^{2}-u_{x}^{2}\right) u_{x y}\right),
$$

the mean curvature of the conjugate harmonic surface, as real and imaginary parts. From the representation (3) of $u$ we get

$$
H+i h=-\left(1+\left|f^{\prime}\right|^{2}\right)^{-3 / 2}\left(f^{\prime}\right)^{2} \overline{f^{\prime \prime}},
$$

hence we see that $H+i h$ satisfies the equation 


$$
\varphi \frac{\partial^{2} \varphi}{\partial z \partial \bar{z}}-\frac{\partial \varphi}{\partial z} \frac{\partial \varphi}{\partial \bar{z}}=0
$$

The last equation is nothing but (9), because of (6). A simple calculation shows that the modulus of any complex-valued solution of (9) is a solution too. Observe, in fact, that the left-hand side of (9) formally agrees with $\varphi^{2}\left((\ln \varphi)_{x x}+(\ln \varphi)_{y y}\right)$. We conclude that

$$
H^{2}+h^{2}
$$

is a further example of a subharmonic function associated with $u$.

The above considerations, together with related strategies, seem to fail if the Gauss curvature $K$ is taken under examination. Although our basic equation (1) is reminiscent of (9), the (negative) term on the right-hand side makes the situation worse. Note that $K$ is not subharmonic (nor superharmonic) in any domain. For instance the (negative of the) Gauss curvature of the harmonic surface

$$
u=\operatorname{Re}(a-i b)^{-1} \exp ((a-i b)(x+i y)+c) \quad(a, b, c=\text { real const. })
$$

has the value

$$
-K=\frac{1}{4}\left(a^{2}+b^{2}\right)(\cosh (a x+b y+c))^{-2}
$$

and achieves its maximum value on the line $a x+b y+c=0$. Another pair is

$$
u=x^{3}-3 x y^{2} \quad-K=36 r^{2}\left(1+9 r^{4}\right)^{-2} ;
$$

here $r^{2}=x^{2}+y^{2},-K$ achieves its minimum at $r=0$ and its maximum on the circle $r=3^{-3 / 4}$.

The main concern of the present paper is to relate

$$
A=\max \{-K(x, y):(x, y) \in \bar{G}\},
$$

the global maximum of $|K|$, with

$$
a=\max \{-K(x, y):(x, y) \in \partial G\},
$$

the maximum of $|K|$ on the boundary. Here $G$ is any domain of the euclidean $(x, y)$-plane such that $K$ is smooth on the closure $\bar{G}$ of $G$.

THEOREM 1. The following alternative holds. Either

$$
A \cdot \operatorname{area}\{(x, y) \in G:-K(x, y)>a\}<\frac{\pi}{p-1},
$$


or

$$
A \leqq \frac{\pi}{p-1}\left[\frac{p}{\pi}\|K\|_{p, \infty}\right]^{p /(p-1)} .
$$

If (14) holds, then

$$
\text { area }\{(x, y) \in G:-K(x, y)>a\} \geqq\left[\frac{p}{\pi}\|K\|_{p, \infty}\right]^{-p /(p-1)}
$$

holds too.

Here $p$ is any number $>1$ and \|\|$_{p, \infty}$ denotes the norm in Lorentz $L(p, \infty)$-space (also called weak $L^{p}$-space), namely

$$
\begin{aligned}
& \|K\|_{p, \infty}=\text { l.u.b. }\left\{C: \int_{E}|K| d x d y<C(\text { area } E)^{1-1 / p}\right. \text { for every } \\
& \text { measurable } E \subset G\} \text {. }
\end{aligned}
$$

Equality holds in (14) if $G$ is the whole of the plane and

$$
K=-C^{2}\left(1+C^{2} r^{2}\right)^{-2} \quad\left(r^{2}=x^{2}+y^{2}, C=\text { const. }\right),
$$

the Gauss curvature of the hyperbolic paraboloid

$$
u=\frac{C}{2}\left(x^{2}-y^{2}\right) \text {. }
$$

Theorem 1 is a corollary of the following.

THEOREM 2. Let

$$
\bar{K}(s)=\frac{1}{s} \text { l.u.b. }\left\{\int_{E}|K| d x d y: E \subset G, \text { area } E=s\right\} .
$$

The following inequality

$$
\frac{1}{\bar{K}(s)} \leqq \frac{1}{A}+\frac{s}{\pi}
$$

holds for any s such that

$$
0 \leqq s \leqq \operatorname{area}\{(x, y) \in G:-K(x, y)>a\} .
$$

Equality holds in (20) for every $s \geqq 0$ if $G$ is the whole of the plane and (17) is valid.

Proof of Theorem 1. From (16) and (19) we get

$$
\|K\|_{p, \infty}=\text { l.u.b. }\left\{s^{1 / p} \bar{K}(s): s>0\right\},
$$


hence (20) yields

$$
(1 / A) s^{-1 / p}+(1 / \pi) s^{1-1 / p} \geqq\|K\|_{p, \infty}^{-1}
$$

for every $s$ that obeys (21). Suppose (13) is violated. Then we can put

$$
s=\frac{\pi}{(p-1) A}
$$

into (23), thus we obtain

$$
p(p-1)^{-1+1 / p} \pi^{-1 / p} A^{-1+1 / p} \geqq\|K\|_{p, \infty}^{-1},
$$

namely inequality (14). Observe that the left-hand side of (25) is exactly the maximum value of the left-hand side of (23). The assertion about the equality sign in (14) follows, thanks to (22). Finally, (15) follows from (14) and the converse of (13).

Let us conclude this section with the following corollary of Theorem 2. Suppose that $G$ is the entire plane $R^{2}$ and $K(x, y) \rightarrow 0$ as $x^{2}+y^{2} \rightarrow \infty$; then

$$
\int_{R^{2}}(-K) d x d y \geqq \pi,
$$

and equality holds in the case (17).

2. A proof of Theorem 2. We can suppose $A>a$, otherwise there is nothing to prove.

Fix any number $t$ such that

$$
a<t<A
$$

and consider the level set

$$
\{(x, y) \in G:-K(x, y)>t\} .
$$

Clearly, (26) is an open nonempty subset of $G$, having a positive distance from the boundary of $G$. The boundary of (26) coincides with

$$
\{(x, y) \in G:-K(x, y)=t\},
$$

provided that the latter is a regular curve, i.e., no critical point of $K$ lies on (27). Sard's theorem tells us that the last-mentioned circumstance occurs for almost every $t$, hence we shall neglect those values of $t$ for which (27) is not the boundary of (26).

Integrating both sides of equation (1) on the level set (26) gives 
(28)

$$
\begin{aligned}
& -t \int_{-K=t}|D K| \sqrt{d x^{2}+d y^{2}}-2 \int_{-K<t}|D K|^{2} d x d y \\
& \quad=8 \int_{-K>t} K^{3} d x d y
\end{aligned}
$$

for the left-hand side of (1) is

$$
\operatorname{div}(K D K)-2|D K|^{2}
$$

and

$$
D K /|D K|
$$

is exactly the outer normal to the boundary of (26). Here $D K$ denotes the gradient of $K$, so that $|D K|=\left(K_{x}^{y}+K_{2}^{2}\right)^{1 / 2}$.

We claim that the following equation

$$
\int_{-K=t}|D K| \sqrt{d x^{2}+d y^{2}}=8 t \int_{-K>t}(-K) d x d y
$$

is a consequence of (28). In fact Federer's coarea formula [6] tells us that the function of $t$, defined for $a<t<A$ by

$$
\Phi(t)=\int_{-K>t}|D K|^{2} d x d y
$$

is absolutely continuous and

$$
\Phi^{\prime}(t)=-\int_{-K=t}|D K| \sqrt{d x^{2}+d y^{2}}
$$

for almost every $t$. In terms of $\Phi$, equation (28) takes the form

$$
-\frac{d}{d t}\left[t^{-2} \Phi(t)\right]=8 t^{-3} \int_{-K>t}(-K)^{3} d x d y,
$$

hence an integration gives

$$
\Phi(t)=8 t^{2} \int_{t}^{A} s^{-3} d s \int_{-K>s}(-K)^{3} d x d y .
$$

The last equation can be rewritten this way

$$
\int_{-K>t}|D K|^{2} d x d y=4 \int_{-K>t}(-K)\left(K^{2}-t^{2}\right) d x d y
$$

after a straightforward use of Fubini's theorem on the right-hand side. Inserting (31) into (28) yields (29).

Let us introduce the distribution function

$$
\mu(t)=\operatorname{area}\{(x, y) \in G:-K(x, y)>t\}
$$

and $K^{*}$, the decreasing rearrangement of $K$ in the sense of Hardy 
and Littlewood. As is well known (see [9], [14], [19], [20]), $K^{*}$ is a kind of inverse function of $\mu$, more precisely, $K^{*}$ is the smallest decreasing function from $[0,+\infty]$ into $[0,+\infty]$ such that

$$
K^{*}(\mu(t)) \geqq t
$$

for every $t \geqq 0$. Equivalently, $K^{*}$ can be defined as the decreasing function from $[0,+\infty]$ into $[0,+\infty]$ such that the level sets $\{s \geqq 0$ : $\left.K^{*}(s)>t\right\}$ are intervals with end points in 0 and $\mu(t)$. The latter statement is summarized by

$$
K^{*}=\int_{0}^{+\infty} \mathbf{1}_{[0, \nu(t)]} d t,
$$

where 1 stands for characteristic function and the integral is taken in the Bochner sense.

The integral at the right-hand side of (29) can be rewritten this way

$$
\int_{-K>t}(-K) d x d y=\int_{0}^{\mu(t)} K^{*}(s) d s,
$$

since both sides of (35) obviously agree with

$$
\int_{t}^{A} s(-d \mu(s)) .
$$

On the other hand, the left-hand side of (29) obeys the following inequality

$$
\int_{-K=t}|D K| \sqrt{d x^{2}+d y^{2}} \geqq 4 \pi \frac{\mu(t)}{-\mu^{\prime}(t)}
$$

for almost every $t$ from $] a, A[$. In fact

$$
\left[\int_{t<-K \leqq t+h}|D K| d x d y\right]^{2} \leqq[\mu(t)-\mu(t+h)]\left[\int_{t<-K \leqq t+h}|D K|^{2} d x d y\right]
$$

for any positive and sufficiently small $h$, because of Schwarz's inequality. Hence

$$
\begin{aligned}
& \text { [length }\{(x, y) \in G:-K(x, y)=t\}]^{2} \\
& \quad \leqq\left[-\mu^{\prime}(t)\right] \int_{-K=t}|D K| \sqrt{d x^{2}+d y^{2}},
\end{aligned}
$$

for $\mu$ is almost everywhere differentiable, (30) holds and

$$
-\frac{d}{d t} \int_{-K>t}|D K| d x d y=\text { length }\{(x, y) \in G:-K(x, y)=t\}
$$

thanks to Federer's coarea formula. Now the standard isoperimetric 
inequality (see e.g., [23]) comes in and gives

$$
\text { [length }\{(x, y) \in G:-K(x, y)=t\}]^{2} \geqq 4 \pi \mu(t) \text {. }
$$

Thus, the wanted inequality (36) follows from (37) and (38).

The triple $(30)(35)(36)$ yields

$$
1 \leqq\left[-\mu^{\prime}(t)\right] \frac{2 t}{\pi \mu(t)} \int_{0}^{\mu(t)} K^{*}(s) d s
$$

for almost every $t$ from the range $a<t<A$.

We claim

$$
-\pi s \frac{d K^{*}}{d s}(s) \leqq 2 K^{*}(s) \int_{0}^{s} K^{*}\left(s^{\prime}\right) d s^{\prime}
$$

for almost every $s$ such that

$$
0<s<\mu(a) \equiv \operatorname{area}\{(x, y) \in G:-K(x, y)>a\} .
$$

In fact, (34) gives

$$
\int_{0}^{+\infty} K^{*}(s) \varphi^{\prime}(s) d s=\int_{0}^{+\infty} \varphi(\mu(t)) d t
$$

for every compactly supported smooth function $\varphi$. Suppose that $\varphi$ is nonnegative and the support of $\varphi$ is concentrated in $] 0, \mu(a)[$. Then (39) and (33) give

$$
\begin{aligned}
& \int_{0}^{+\infty} \varphi(\mu(t)) d t \\
& \quad \leqq \int_{0}^{+\infty} \varphi(\mu(t)) \frac{2 K^{*}(\mu(t))}{\pi \mu(t)}\left[\int_{0}^{\mu(t)} K^{*}(s) d s\right]\left(-\mu^{\prime}(t)\right) d t,
\end{aligned}
$$

hence we have

$$
\int_{0}^{+\infty} K^{*}(s) \varphi^{\prime}(s) d s \leqq \int_{0}^{+\infty} \varphi(s) \frac{2 K^{*}(s)}{\pi s}\left[\int_{0}^{s} K^{*}\left(s^{\prime}\right) d s^{\prime}\right] d s,
$$

since $\int \cdots\left(-\mu^{\prime}(t)\right) d t \leqq \int \cdots(-d \mu(t))$. As $K^{*}$ is absolutely continuous (see [25] for instance), inequality (40) follows.

A convenient device for discussing inequality (40a) is to use (19). One may already know or easily check (see e.g., [14], [19], [20]) that $\bar{K}$ is the maximal function associated with $K^{*}$, namely

$$
\bar{K}(s)=\frac{1}{s} \int_{0}^{s} K^{*}\left(s^{\prime}\right) d s^{\prime} .
$$

In terms of $\bar{K}$, (40a) reads as follows 


$$
-\frac{d}{d s}\left[\pi s^{2} \frac{d \bar{K}}{d s}(s)\right] \leqq \frac{d}{d s}[s \bar{K}(s)]^{2} .
$$

Hence $\pi s^{2}(d \bar{K}(s) / d s)+(s \bar{K}(s))^{2}$ is an increasing function of $s$. As such function vanishes at $s=0$ (note that $\bar{K}(s) \rightarrow K^{*}(0)=A$, $s(d \bar{K}(s) / d s)=K^{*}(s)-\bar{K}(s) \rightarrow 0$ as $\left.s \rightarrow 0\right)$, we must have

$$
\pi \frac{d \bar{K}}{d s}(s)+(\bar{K}(s))^{2} \geqq 0,
$$

that is

$$
\frac{d}{d s} \frac{1}{\bar{K}(s)} \leqq \frac{1}{\pi} .
$$

Inequality (43) holds for $s$ in the range (40b). Integrating (43) gives the wanted conclusion (20), provided $s$ is restricted by (40b) and the formula

$$
\bar{K}(0)=K^{*}(0)=A
$$

is used again.

When does the formula

$$
\bar{K}(s)=\left(\frac{1}{A}+\frac{s}{\pi}\right)^{-1}
$$

hold for every $s$ ? A glance at the previous arguments shows that (45) holds if, and only if, equality holds for almost every $t$ both in isoperimetric inequality (38) and in (37). Equality in (38) leads to the conclusion that: (i) every level set (26) is a disk. Equality in (37) holds if and only if: (ii) $|D K|$, the magnitude of $D K$, is constant along almost every level line (27). Properties (i) and (ii) are valid if (and actually imply that, as the arguments from [1] should show) $K$ is circularly symmetric, namely

$$
-K(x, y)=f(r),
$$

and $f$ decreases as $r$ increases. Here $r$ is the distance from some fixed point, $r=\sqrt{x^{2}+y^{2}}$ say.

Let us lock for those solutions of equation (1), that have the form (46) (circular weaves). We find the following equation

$$
r f f^{\prime \prime}-r\left(f^{\prime}\right)^{2}+f f^{\prime}+8 r(f)^{3}=0 \quad('=d / d r),
$$

whose general solution (cfr. [15], part 3, Chapter 6, ex. no. 172) is

$$
f(r)=\left(C n r^{n-1}\right)^{2}\left(1+C^{2} r^{2 n}\right)^{-2} \quad(C, n=\text { const. } \geqq 0) .
$$

Functions $K$, given by (46) (47), actually are weak solutions 
(i.e., solutions in the sense of distributions) to equation (1) if and only if $n \geqq 1$. Incidentally, these solutions are not smooth if $n$ is not an integer. A decreasing function of $r$ is gotten from (47) only by choosing $n=1$. Note that the negative of (47) is the Gauss curvature of the harmonic surface

$$
u=\frac{C}{n+1} \operatorname{Re}(x-i y)^{n+1} .
$$

In conclusion, the pair

$$
K=-C^{2}\left(1+C^{2} r^{2}\right)^{-2} \quad u=\frac{C}{2} \operatorname{Re}(x+i y)^{2}
$$

must lead to formula (45).

Our theorem is fully proved.

3. Minimal surfaces. Estimates for the Gauss curvature of minimal surfaces have been given by several authors. Heinz [10] proved that, if $u$ is a solution to the minimal surfaces equation

$$
\left(1+u_{y}^{2}\right) u_{x x}-2 u_{y} u_{x} u_{x y}+\left(1+u_{x}^{2}\right) u_{y y}=0
$$

in a disk $x^{2}+y^{2}<R^{2}$, then the Gauss curvature of $K$ of the graph of $u$ (cfr. formula (2)) satisfies

$$
|K(0,0)| \leqq c \cdot R^{-2},
$$

where $c$ is an absolute constant. Finn and Osserman [8] proved that the best constant in (51) satisfies $\left(\pi^{2} / 2\right) \leqq c<6$, and (51) holds with $c=\pi^{2} / 2$ if the gradient of $u$ vanishes at the origin. Other improvements on Heinz' result have been proposed by E. Hopf [11] and J. C. C. Nitsche [18]. For instance, Nitsche proved that (51) can be replaced by

$$
|K(0,0)| \leqq \frac{49}{4} R^{-2}\left[1+u_{x}^{2}(0,0)+u_{y}^{2}(0,0)\right]^{-2} .
$$

Further generalizations are in Osserman [21]. See [22], §11, for a discussion on this matter. Let us mention that estimates for the Gauss curvature of surfaces, having a constant mean curvature, are in Spruck [24].

In this section we briefly discuss the following theorem.

THEOREM 3. Let $M$ be an embedded minimal surface in euclidean 3-space and let $K$ be the Gauss curvature of $M$. The following inequality 


$$
\frac{1}{\bar{K}(s)} \leqq \frac{1}{A}+\frac{s}{2 \pi}
$$

holds for every s such that

$$
0 \leqq s \leqq H_{2}\{x \in M:|K(x)|>a\}
$$

Here

$$
\begin{gathered}
\bar{K}(s)=\frac{1}{s} \text { l.u.b. }\left\{\int_{E}|K(x)| H_{2}(d x): E \subset M, H_{2}(E)=s\right\} \\
A=\text { l.u.b. }\{|K(x)|: x \in M\} \\
a=\text { l.u.b. }\{|K(x)|: x \in \partial M\},
\end{gathered}
$$

and $H_{2}$ denotes the two-dimensional Hausdorff measure.

REMARKS. (i) Theorem 3 yields a number of estimates for the Gauss curvature of minimal surfaces. Such estimates are the exact analogs of those presented in Theorem 1 (but are omitted here for the sake of brevity). Note that Theorem 3 involves rearrangements of the curvature on the surface itself. This is the main difference between Theorems 2 and 3 . Note that in the present context formulas (33) (34) (41) still match (55), while formula (32) for the distribution function must be replaced by

$$
\mu(t)=H_{2}\{x \in M:|K(x)|>t\} .
$$

(ii) Whether equality in (53) may actually be achieved by some minimal surface is not discussed here. However, a minimal surface can easily be shown such that the power series expansion of $1 / \bar{K}(s)$ near $s=0$ begins with the right-hand side of (53). A surface having this property is Enneper. As is well known (see e.g., [5], $\S \S 3-5)$, the surface of Enneper has the following parametric representation

$$
x_{1}=C u\left(1-\frac{u^{2}}{3}+v^{2}\right) \quad x_{2}=C v\left(1+u^{2}-\frac{v^{2}}{3}\right) \quad x_{3}=C\left(u^{2}-v^{2}\right),
$$

hence the following metric

$$
d x_{1}^{2}+d x_{2}^{2}+d x_{3}^{2}=C^{2}\left(1+u^{2}+v^{2}\right)^{2}\left(d u^{2}+d v^{2}\right)
$$

and the curvature

$$
K=-4 C^{-2}\left(1+u^{2}+v^{2}\right)^{-4} .
$$

Here $C$ is any real constant, that is related to the maximum $A$ of $|K|$ by: $4 C^{-2}=A$. Using (58) one finds 


$$
\begin{aligned}
\mu(t) & =\int_{C^{2}\left(1+u^{2}+v^{2}\right)^{4}<4 / t} C^{2}\left(1+u^{2}+v^{2}\right) d u d v \\
& =\frac{4 \pi}{3} A^{-1 / 4}\left[t^{-3 / 4}-A^{-3 / 4}\right],
\end{aligned}
$$

hence from (41) and (34) one gets

$$
\frac{1}{\bar{K}(s)}=\frac{s}{4 \pi}\left[1-\left(1+\frac{3 A}{4 \pi} s\right)^{-1 / 3}\right]^{-1}=\frac{1}{A}+\frac{s}{2 \pi}+\cdots .
$$

(iii) Some assumptions on the topology of $M$ should be made for Theorem 3 to hold in the form stated. For instance, we might assume that $M$ is simply connected. Double connectivity might also suffice. As a matter of a fact, Theorem 3 holds if the level sets of the curvature

$$
\{x \in M:|K(x)|>t\}
$$

obey the isoperimetric inequality

$$
(\text { perimeter) })^{2} \geqq 4 \pi \text { (area) }
$$

whenever $t$ exceeds the maximum $a$ of $|K|$ on $\partial M$. Theorems asserting the validity of isoperimetric inequality (60) for subdomains of minimal surfaces are in Carleman [4], Beckenbach-Radó [2], Huber [13], Hsiung [12], Feinberg [7]. See [23], Theorems 4.2 and 5.3.

Our proof of Theorem 3 is entirely analogous to that of Theorem 2 and consists of a suitable treatment of a special partial differential equation. The only change is that we are now faced by a partial differential equation on a manifold.

The Gauss curvature $K$ of a smoothly embedded surface $M$ in euclidean 3-space satisfies

$$
K \Delta K-|D K|^{2}=4 K^{3}
$$

if and only if $M$ is either minimal or locally isometric to a minimal surface. See e.g., [3], vol. 1, §211. Equation (61) holds on the surface itself; that is $D$ and $\Delta$ are the covariant derivative (or intrinsic gradient) and the Laplace-Beltrami operator on $M$. If $u, v$ are local coordinates on $M$ and

$$
E d u^{2}+2 F d u d v+G d v^{2}
$$

defines the metric tensor on $M$, one has

$$
g|D \varphi|^{2}=G\left(\frac{\partial \varphi}{\partial u}\right)^{2}-2 F \frac{\partial \varphi}{\partial u} \frac{\partial \varphi}{\partial v}+E\left(\frac{\partial \varphi}{\partial v}\right)^{2}
$$




$$
\sqrt{g} \Delta \varphi=\frac{\partial}{\partial u} \frac{1}{\sqrt{g}}\left(G \frac{\partial \varphi}{\partial u}-F \frac{\partial \varphi}{\partial v}\right)+\frac{\partial}{\partial v} \frac{1}{\sqrt{g}}\left(-F \frac{\partial \varphi}{\partial u}+E \frac{\partial \varphi}{\partial v}\right)
$$

for every smooth scalar field $\varphi$ on $M$. As usual, $g$ denotes $E G-F^{2}$. Incidentally, a proof of (61), in the case where the relevant surface is minimal, is easily obtained from the Weierstrass representation. In fact, let a minimal surface have the parametric representation

$$
x_{k}=\operatorname{Re} \int^{u+i v} \varphi_{k}(\zeta) d \zeta \quad(k=1,2,3)
$$

where

$$
\varphi_{1}=\frac{1}{2} f\left(1-h^{2}\right) \quad \varphi_{2}=\frac{i}{2} f\left(1+h^{2}\right) \quad \varphi_{3}=f h,
$$

$f$ is holomorphic, $h$ is meromorphic, and $f h^{2}$ is free from poles (cfr. e.g., [22], Lemma 8.2). Then $u, v$ are isothermal coordinates; the coefficients of the first quadratic form satisfy

$$
E=G=\sqrt{g}=\frac{1}{4}|f|^{2}\left(1+|h|^{2}\right)^{2}, \quad F=0 ；
$$

we have the formula

$$
K=-16|f|^{-2}\left|h^{\prime}\right|^{2}\left(1+|h|^{2}\right)^{-4}
$$

for the Gauss curvature of our surface. As in the proof of Lemma 1 , one can check that any expression $K$ of the form (66b) satisfies

$$
K \frac{\partial^{2} K}{\partial \zeta \partial \bar{\zeta}}-\frac{\partial K}{\partial \zeta} \frac{\partial K}{\partial \bar{\zeta}}=\sqrt{g} K^{3},
$$

where $\zeta$ denotes the complex variable $u+i v$ and $g$ is given by (66a). The last equation is nothing but (61), thanks to (63) (64) and $(66 a)$.

Let us sketch a proof of Theorem 3. The basic steps are as follows.

(i) An integration of both sides of equation (61) over the level set (59) gives

$$
\begin{aligned}
t \int_{-K=t}|D K| H_{1}(d x) & +2 \int_{-K>t}|D K|^{2} H_{2}(d x) \\
& +4 \int_{-K>t} K^{3} H_{2}(d x)=0,
\end{aligned}
$$

for almost every $t$ such that $a<t<A$. Here $H_{1}$ stands for 1-dimensional Hausdorff measure. 
(ii) The coarea formula [6] gives

$$
\frac{d}{d t} \int_{-K>t}|D K|^{2} H_{2}(d x)=-\int_{-K=t}|D K| H_{1}(d x)
$$

for almost every $t$ such that $a<t<A$.

(iii) From the previous steps we get the formula

$$
\int_{-K=t}|D K| H_{1}(d x)=4 t \int_{-K>t}(-K) H_{2}(d x),
$$

where $a<t<A$.

(iv) Formulas (58) and (34) give

$$
\int_{-K>t}(-K) H_{2}(d x)=\int_{0}^{\mu(t)} K^{*}(s) d s .
$$

(v) The formula from step (ii), Schwarz inequality and formula (58) give

$$
\left[H_{1}\{x \in M:-K(x)=t\}\right]^{2} \leqq-\mu^{\prime}(t) \cdot \int_{-K=t}|D K| H_{1}(d x),
$$

provided $a<t<A$.

(vi) The isoperimetric inequality (60) tells us that the left-hand side of the inequality from step ( $v$ ) is estimated from below by $4 \pi \mu(t)$.

(vii) A collation of previous steps gives

$$
\pi \mu(t) \leqq\left[-\mu^{\prime}(t)\right] t \cdot \int_{0}^{\mu(t)} K^{*}(s) d s,
$$

for almost every $t$ such that $a<t<A$.

The conclusion follows from step (vii), via the same argument we used in the discussion of inequality (39).

Added in proof. Prof. R. Osserman has pointed out that the inequality we have stated in the corollary of Theorem 2 can be derived from the representation (5) of $K$ (provided the relevant harmonic function $u$ is not linear), without using any assumption on the behaviour of $K$ at infinity. We are indebted to him for drawing our attention to his paper Some geometric properties of polynomial surfaces (Comm. Math. Helvetici, 37 (1963), where somewhat related results are proved.

\section{REFERENCES}

1. G. Aronsson and G. Talenti, Estimating the integral of a function in terms of a distribution function of its gradient, Boll. Unione Mat. Italiana, 18-B (1981). 
2. E. F. Beckenbach and T. Radó, Subharmonic functions and surfaces of negative curva. ture, Trans. Amer. Math. Soc., 35 (1933).

3. L. Bianchi, Lezioni di geometria differenziale, Bologna, 1927.

4. T. Carleman, Zur theorie der Minimalfä̈chen, Math. Z., 9 (1921).

5. M. Do Carmo, Differential Geometry of Curves and Surfaces, Prentice Hall, 1976.

6. H. Federer, Curvature measures, Trans. Amer. Math. Soc., 93 (1959).

7. J. M. Feinberg, The isoperimetric inequality for doubly-connected minimal surfaces in $R^{n}$, J. d'Analyse Math., 32 (1977).

8. R. Finn and R. Osserman, On the Gauss curvature of nonparametric minimal sur. faces, J. d'Analyse Math., 12 (1964).

9. Hardy, Littlewood and Pólya, Inequalities, Cambridge Univ. Press, 1964.

10. E. Heinz, Über die Lösungen der Minimalfä̈chengleichung, Nachrichten der Akad. d. Wiss. in Göttingen, Math. Phys. Klasse, no. 8, 1952.

11. E. Hopf, On an inequality for minimal surfaces $z=z(x, y)$, J. Rational Mech. Anal., 2 (1953).

12. C. C. Hsiung, Isoperimetric inequalities for two-dimensional Riemannian manifolds with boundary, Ann. of Math., 73 (1961).

13. A. Huber, On the isoperimetric inequality on surfaces of variable Gaussian curvature, Ann. of Math., 73 (1961).

14. R. Hunt, On $L(p, q)$ spaces, Ein segnement Math., 12 (1966).

15. E. Kamke, Differentialgleichungen I, Leipzig, 1959.

16. J. Liouville, Sur le théorème de M. Gauss, concernant le produit des deux rayons de courbure principaux en chaque point d'une surface, Notes de J. Liouville a l'ouvrage Ap. plications de l'Analyse a la Géometrie par G. Monge, Paris, Bachelier Ed., 1850.

17. Sur l'équation aux différences partielles $d^{2} \log \lambda / d u d v \pm \lambda / 2 a^{2}=0$, J. de Mathématiques Pures et Appliqués, 18 (1853).

18. J. C. C. Nitsche, On an estimate for the curvature of minimal surfaces $z=z(x, y), \mathrm{J}$. Math. Mech., 7 (1958).

19. R. O'Neil, Convolution operators and L $(p, q)$ spaces, Duke Math. J., 301963.

20 . Integral transforms and tensor products on Orlicz spaces and $L(p, q)$ spaces, J. d'Analyse Math., 21 (1968).

21. R. Osserman, On the Gauss curvature of minimal surfaces, Trans. Amer. Math. Soc., 96 (1960).

22. - A Survey of Minimal Surfaces, Van Nostrand, 1969.

23. The isoperimetric inequality, Bull. Amer. Math. Soc., 84 (1978).

24. J. Spruck, Gauss curvature estimates for surfaces of constant mean curvature, Comm. Pure Appl. Math., 27 (1974).

25. G. Talenti, Best constant in Sobolev inequality, Ann. Mat. Pura Appl., 110 (1976).

26. - Nonlinear elliptic equations, rearrangements of functions and Orlicz spaces, Ann. Mat. Pura Appl., 120 (1979).

Received February 2, 1981.

Istituto Matematico dell'Università

viale Morgagni $67 \mathrm{~A}$

50134 FIRENZE, ITALY 


\section{PACIFIC JOURNAL OF MATHEMATICS}

\section{EDITORS}

DONALD BABBITT (Managing Editor)

University of California

Los Angeles, CA 90024

Hugo Rossi

University of Utah

Salt Lake City, UT 84112

C. C. MOore and Arthur Agus

University of California

Berkeley, CA 94720
J. DugundJI

Department of Mathematics

University of Southern California

Los Angeles, CA 90007

R. FinN and J. MILGRAM

Stanford University

Stanford, CA 94305

\section{ASSOCIATE EDITORS}
R. ARENS
E. F, BECKENBACH
B. H. NeumanN
F. WOLF
K. YoSHIDA

\section{SUPPORTING INSTITUTIONS}

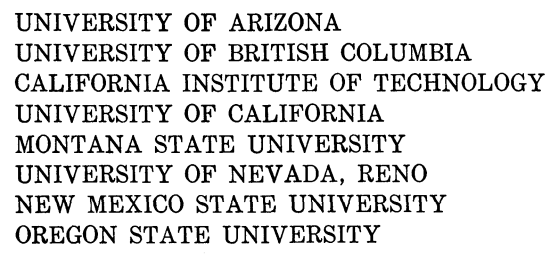

UNIVERSITY OF ARIZONA

UNIVERSITY OF BRITISH COLUMBIA

CALIFORNIA INSTITUTE OF TECHNOLOGY

UNIVERSITY OF CALIFORNIA

MONTANA STATE UNIVERSITY

UNIVERSITY OF NEVADA, RENO

NEW MEXICO STATE UNIVERSITY

OREGON STATE UNIVERSITY

\author{
UNIVERSITY OF OREGON \\ UNIVERSITY OF SOUTHERN CALIFORNIA \\ STANFORD UNIVERSITY \\ UNIVERSITY OF AAWAII \\ UNIVERSITY OF TOKYO \\ UNIVERSITY OF UTAH \\ WASHINGTON STATE UNIVERSITY \\ UNIVERSITY OF WASHINGTON
}

The Supporting Institutions listed above contribute to the cost of publication of this Journal, but they are not owners or publishers and have no responsibility for its content or policies,

Mathematical parers intended for publication in the Pacific Journal of Mathematics should be in typed form or offset-reproduced, (not dittoed), double spaced with large margins. Please do not use built up fractions in the text of the manuscript. However, you may use them in the displayed equations. Underline Greek letters in red, German in green, and script in blue. The first paragraph or two must be capable of being used separately as a synopsis of the entire paper. Please propose a heading for the odd unmbered pages of less than 35 characters. Manuscripts, in triplicate, may be sent to any one of the editors. Please classify according to the scheme of Math. Reviews, Index to Vol. 39. Supply name and address of author to whom proofs should be sent. All other communications should be addressed to the managing editor, or Elaine Barth, University of California, Los Angeles, California, 90024.

50 reprints to each author are provided free for each article, only if page charges have been substantially paid. Additional copies may be obtained at cost in multiples of 50 .

The Pacific Journal of Mathematics is issued monthly as of January 1966, Regular subscription rate: $\$ 114.00$ a year $(6$ Vol., 12 issues). Special rate: $\$ 57.00$ a year to individual members of supporting institution.

Subscriptions, orders for numbers issued in the last three calendar years, and changes of address shoud be sent to Pacific Journal of Mathematics, P.O. Box 969, Carmel Valley, CA 93924, U.S.A. Old back numbers obtainable from Kraus Periodicals Co., Route 100, Millwood, NY 10546.

PUBLISHED BY PACIFIC JOURNAL OF MATHEMATICS, A NON-PROFIT CORPORATION

Printed at Kokusai Bunken Insatsusha (International Academic Printing Co., Ltd.). 8-8, 3-chome, Takadanobaba, Shinjuku-ku, Tokyo 160, Japan.

Copyright (C) 1982 by Pacific Journal of Mathematics Manufactured and first issued in Japan 


\section{Pacific Journal of Mathematics}

\section{Vol. 101, No. 2 December, 1982}

Jean Bourgain, A Hausdorff-Young inequality for $B$-convex Banach

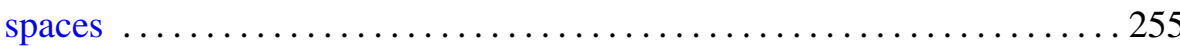

J. L. Brenner and Lorraine L. Foster, Exponential Diophantine

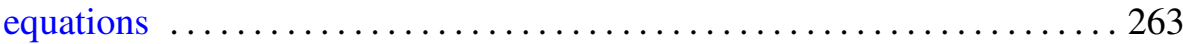

Henry H. Glover and William Duncan Homer, II, Fixed points on flag

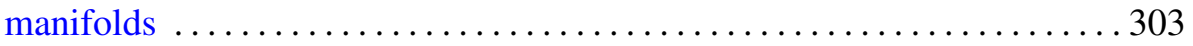

Lothar Hahn, A note on stochastic methods in connection with approximation theorems for positive linear operators $\ldots \ldots \ldots \ldots \ldots . \ldots 307$

James P. Henderson, Approximating cellular maps between low-dimensional polyhedra

V. K. Jain, Certain transformations of basic hypergeometric series and their

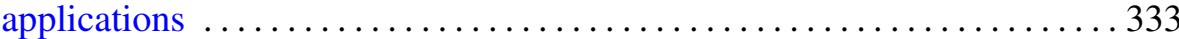

Charles David Keys, On the decomposition of reducible principal series

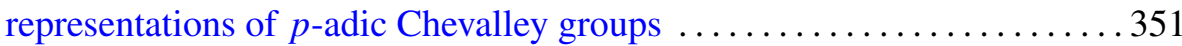

M. S. Klamkin and A. Meir, Ptolemy's inequality, chordal metric,

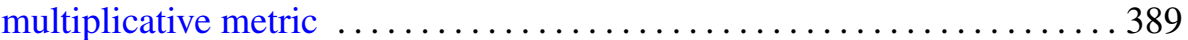

Robert F. Lax, Independence of normal Weierstrass points under

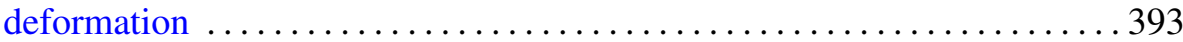

Leonid A. Luxemburg, On compactifications of metric spaces with

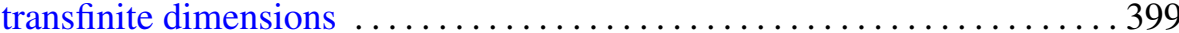

Carlton James Maxson, Martin Ross Pettet and Kirby C. Smith, On semisimple rings that are centralizer near-rings .....

Teodor C. Przymusiński, Extending functions from products with a metric factor and absolutes

Giorgio Talenti, A note on the Gauss curvature of harmonic and minimal surfaces

D. M. Terlinden, A spectral containment theorem analogous to the semigroup theory result $e^{t \sigma(A)} \subseteq \sigma\left(e^{t A}\right)$ 